\title{
Variação química no óleo essencial das folhas de seis indivíduos de Duguetia furfuracea (Annonaceae)
}

\author{
Janaina L. Valter, ${ }^{1}$ Karina M. C. Alencar, ${ }^{1}$ Angela L. B. Sartori, ${ }^{1}$ Evandro A. Nascimento, ${ }^{2}$ \\ Roberto Chang, ${ }^{2}$ Sérgio A. L. de Morais, ${ }^{2}$ Valdemir A. Laura, ${ }^{3}$ Nídia C. Yoshida, ${ }^{4}$ Carlos A. \\ Carollo, ${ }^{4}$ Denise B. da Silva, ${ }^{4}$ Rafaela F. Grassi, ${ }^{4}$ João R. Fabri, ${ }^{4}$ João M. de Siqueira ${ }^{*, 4}$
}

\author{
${ }^{1}$ Departamento de Biologia, Centro de Ciências Biológicas e da Saúde, Universidade Federal de Mato Grosso do \\ Sul, C.P. 549, 79070-900 Campo Grande-MS, Brasil, \\ ${ }^{2}$ Instituto de Química, Universidade Federal de Uberlândia, C.P. 593, Av. João Naves de Ávila, 2121, Campus \\ Santa Mônica, 38400-089 Uberlândia-MG, Brasil, \\ ${ }^{3}$ Empresa Brasileira de Pesquisa Agropecuária, Embrapa Gado de Corte, C.P. 154, 79002-970 Campo Grande- \\ MS, Brasil, \\ ${ }^{4}$ Departamento de Farmácia, Centro de Ciências Biológicas e da Saúde, Universidade Federal de Mato Grosso \\ do Sul, C.P. 549, 79070-900 Campo Grande-MS, Brasil
}

\begin{abstract}
RESUMO: Duguetia furfuracea, Annonaceae, uma invasora de pastagens, é também usada na medicina tradicional em diversos estados no Brasil. Devido à significante diferença de odor entre dois grupos de indivíduos desta planta em um remanescente de cerrado em Campo Grande, Mato Grosso do Sul, seis amostras foram selecionadas de acordo com a intensidade de odor em suas folhas: In-01, In-03 e In-05: odor pronunciado (Perfil A); In-02, In-04 e In-06: odor fraco ou ausência de odor (Perfil B). As folhas foram coletadas e submetidas a hidrodestilação para obtenção dos óleos essenciais, os quais foram analisados por CG/EM. Uma boa compatibilidade foi encontrada entre os resultados obtidos por CG/EM e a análise olfativa adotada para as amostras: espécimes odor pronunciado apresentaram uma alta porcentagem de monoterpenos e alguns sesquiterpenos: In-01 ( $\beta$-felandreno, 42,2\%; mirceno, 6,8\%; $\alpha$-felandreno, 4,6\%); In-03 (terpin4-ol, 21,6\%; sabineno, 17,3\%; $p$-cimeno, 5,6\%); In-05 (sabineno, 25,1\%; terpin-4-ol, 16,2\%; $p$ cimeno, $8,3 \%$ ). Apenas sesquiterpenos foram encontrados nos espécimes que apresentaram odor fraco ou ausência do mesmo (com constituinte majoritário biciclogermacreno: 21,4\%, 24,0\%, and $29,1 \%$, respectivamente, para os In-02, In-04 e In-06).
\end{abstract}

Unitermos: Duguetia furfuracea, Annonaceae, óleo volátil.

\begin{abstract}
Chemical variability of the essential oil of the leaves from six individuals of Duguetia furfuracea (Annonaceae)". Duguetia furfuracea, Annonaceae, a pasture weed, is also used in folk medicine in several Brazilian states. Because of the significant difference in odor between two groups of this plant in a remnant patch of savanna in Campo Grande county, Mato Grosso do Sul, six samples were selected according to the odor intensity in the leaves (In-01, In-03, and In-05: pronounced odor; In-02, In-04, and In-06: weak odor or its absence). The leaves were collected and subjected to steam distillation for extraction of essential oils, which were analyzed by GC-MS. A good agreement was found between CG/MS results and olfactory evaluation of the samples: specimens exhibiting leaf scent had a high percentage of monoterpenes and some sesquiterpenes, as follows: In-01 ( $\beta$-phellandrene, 42.2\%; myrcene, $6.8 \%$; $\alpha$-phellandrene, 4.6\%); In-03 (terpin-4-ol, 21.6\%; sabinene, $17.3 \%$; $p$-cymene, $5.6 \%$ ); In05 (sabinene, 25.1\%; terpin-4-ol, 16.2\%; p-cymene, 8.3\%). Only sesquiterpenes were found in the specimens having weakly scented or scentless leaves (main constituent bicyclogermacrene: $21.4 \%, 24.0 \%$, and $29.1 \%$, respectively, for In-02, In-04 and In-06).
\end{abstract}

Keywords: Duguetia furfuracea, Annonaceae, volatile oil.

\section{INTRODUÇÃO}

Duguetia furfuracea (Annonaceae), um arbusto típico de cerrados secos do Brasil Central é encontrada em regiões de pastagens brasileiras, sendo particularmente freqüente em Mato Grosso do Sul, Mato Grosso, Goiás e Minas Gerais. A espécie é perene, ereta, arbustiva e muito ramificada (Lorenzi, 2000), com base subterrânea que a torna resistente ao fogo (Pott \& Pott, 1994; Mass et al., 2001). É freqüentemente encontrada 
em áreas do cerrado sul-mato-grossense que, por ação antropogênica, foram transformadas em pastagens. Por sua alta capacidade de crescimento vegetativo, suas grandes infestações são de difícil controle (Lorenzi, 2000).

Popularmente conhecida como "araticumseco", conta com algumas citações na medicina popular, como a de que suas sementes têm uso parasiticida, especialmente contra pediculose (SiberbauerGottsberger, 1981/1982; Agra et al., 2007). A infusão de pequenos ramos e folhas dessa espécie é indicada para reumatismo (Rodrigues \& Carvalho, 2001) e cápsulas contendo suas folhas são apontadas como úteis no tratamento de disfunção renal, havendo o reconhecimento de direito de patente já sido requerido (Da Silva Coelho, 2003).

Do ponto de vista químico, a espécie tem sido nosso tema de investigação. Em trabalhos desenvolvidos recentemente com um exemplar selecionado em um remanescente de cerrado em Campo Grande, MS, o óleo volátil presente nas folhas foi submetido ao fracionamento fitoquímico usual e seis sequiterpenos foram isolados e identificados (Carollo et al., 2005) e foram obtidos também diferentes extratos das folhas e ramos; a partir desses extratos foram isolados um flavonóide e alcalóides, que foram testados contra atividade tripanossomicida (Carollo et al., 2006a). Além disso, dois alcalóides contendo uma funcionalização $N$-nitroso foram isolados e descritos (Carollo et al., 2006b). Em estudo realizado anteriormente com 31 espécies de Annonaceae, foram identificados nas folhas de $D$. furfuracea os seguintes flavonóides glicosilados: 3-O-galactosilgalactosil-kaempferol, 3-O-galactosilisoramnetina, 3-O-galactosilramnosil-isoramnetina e 3-O-ramnosilglucosil-isoramnetina (Santos \& Salatino, 2000).

O presente trabalho teve como objetivo obter informações sobre a composição química do óleo essencial de seis indivíduos de D. furfuracea previamente selecionados do mesmo remanescente de cerrado citado. Para isso, foi feito o estudo da variação qualitativa e quantitativa do óleo essencial obtido das folhas.

\section{MATERIAL E MÉTODOS}

\section{Seleção dos indivíduos por análise sensorial}

Foram selecionados seis indivíduos de $D$. furfuracea, a partir uma população existente em um remanescente de cerrado localizado em Campo Grande, MS. O critério de seleção e de coleta foi a impressão de odor constatada pelos pesquisadores do presente trabalho, lotados na UFMS, que após maceração de folhas de cada individuo, era seguida de avaliação olfativa. Esse procedimento permitiu dividir a população em dois grupos distintos. A análise sensorial (classificação do aroma das

folhas) foi desenvolvida como descrita por Franco et al. (2004). Os grupos foram designados como "perfil A" e "perfil B", com base nas impressões de odor (Ngassum et al., 2004): os indivíduos de perfil A foram aqueles que proporcionaram uma impressão olfativa acentuada (In01, 03 e 05); os de perfil B proporcionaram impressão menos acentuada ou nenhuma impressão (In- 02, 04 e 06). Esse foi o único critério de avaliação utilizado, não sendo posteriormente aplicado nenhum protocolo de avaliação mais detalhado, tal como o "Odour Activity Value" (OAV) (Grosch, 1998, 2001).

Os indivíduos foram coletados (distância mínima de $3 \mathrm{~m}$ e máxima de $100 \mathrm{~m}$ ) e as exsicatas foram identificadas pelo Prof. Dr. R. Mello-Silva e depositadas no Herbário CGMS, da UFMS, sob números 11951 (In01), 11952 (In-02), 11953 (In-03), 11954 (In-04), 11955 (In-05) e 11956 (In-06).

\section{Análises sensorial e morfológica dos indivíduos}

Os caracteres analisados foram: (a) altura, (b) comprimento e largura das folhas, (c) presença e tipo de tricomas, (d) presença e posição de galhas, (e) coloração e tamanho das sépalas e pétalas. A terminologia adotada para classificar os tricomas foi elaborada de acordo com o proposto na literatura (Harris \& Harris, 1994).

\section{Coleta, obtenção e análise do óleo essencial das folhas}

\section{Extração do óleo essencial}

Foram realizadas duas coletas distintas das folhas dos seis indivíduos para extração do óleo essencial: a primeira em maio de 2004, no período da manhã (entre $7 \mathrm{~h} \mathrm{e} 8 \mathrm{~h}$ ) em um mesmo dia, e a segunda em novembro de 2004, no mesmo intervalo de horários. A extração do óleo essencial das folhas frescas (100 g) foi realizada por hidrodestilação em aparelho de Clevenger segundo técnica usual (Barbosa-Filho et al., 2008) e todas as extrações foram desenvolvidas durante $4 \mathrm{~h}$. Somente a primeira coleta (outono de 2004) foi analisada por CG/EM, CCD e análise de aroma. Não houve padronização das folhas a serem colhidas, sendo coletadas folhas de todo o indivíduo

\section{Análise das amostras de óleos essenciais por cromatografia gasosa e espectrometria de massa}

As amostras de óleos essenciais (coleta de maio de 2004) foram analisadas por cromatografia gasosa (CG) em equipamento Shimadzu modelo 17A, equipado com detector de ionização de chama de hidrogênio e coluna capilar SBP-5 (30 cm de comprimento, 0,25 mm de diâmetro interno, $0,25 \mu \mathrm{m}$ de espessura de filme). $\mathrm{O}$ gás de arraste utilizado foi o nitrogênio. A temperatura inicial da coluna foi de $60{ }^{\circ} \mathrm{C}$, sendo programada para 
acréscimos de $3{ }^{\circ} \mathrm{C} / \mathrm{min}$ até se atingir um máximo de $240{ }^{\circ} \mathrm{C}$. As temperaturas do injetor e do detector foram fixadas em $220{ }^{\circ} \mathrm{C}$ e $240{ }^{\circ} \mathrm{C}$, respectivamente. Uma quantidade de $10 \mathrm{mg}$ de cada amostra foi diluída em 1 $\mathrm{mL}$ de pentano, sendo injetado $1 \mu \mathrm{L}$ dessa solução.

Os compostos foram identificados por cromatografia gasosa acoplada a espectrômetro de massas (CG/EM), em equipamento Shimadzu modelo CG 17A, com detector seletivo de massas modelo QP 5000 (Shimadzu). A coluna cromatográfica utilizada foi do tipo capilar de sílica fundida com fase estacionária DB-5, de $30 \mathrm{~mm}$ de comprimento, 0,25 $\mathrm{mm}$ de diâmetro interno e $0,25 \mu \mathrm{m}$ de espessura de filme, utilizando hélio como gás carreador. As temperaturas foram de $220{ }^{\circ} \mathrm{C}$ no injetor e $300{ }^{\circ} \mathrm{C}$ no detector. A temperatura do forno foi programada para variar de $60{ }^{\circ} \mathrm{C}$ a $240{ }^{\circ} \mathrm{C}$, com acréscimo de $3{ }^{\circ} \mathrm{C} / \mathrm{min}$.

As identificações foram feitas por meio das espectroteca Wiley (140, 229 e 338) e por índices de Kovat (Adams, 1995).

\section{RESULTADOS E DISCUSSÃO}

Os seguintes rendimentos em porcentagem de volume de óleo essencial por peso de planta fresca $(\% \mathrm{v} / \mathrm{p})$ foram observados (\% outono/\% primavera): In-01, $0,8 / 0,9$; In-02, 0,6/0,7; In-03, $0,5 / 0,8$; In-04, $0,3 / 0,3$; In-05, 0,6/0,5; In-06, 0,5/0,5. Em alguns indivíduos observa-se um discreto aumento do volume de óleo essencial, mas não foi possível relacionar com os caracteres morfológicos descritos na Tabela 2 e tampouco com o período sazonal.

A composição do óleo essencial das folhas dos indivíduos coletados em maio de 2004 e analisada por CG/EM é apresentada na Tabela 1. Os monoterpenos perfizeram $65,6 \%, 57,4 \%$ e $68,6 \%$, respectivamente, do teor total constituído no óleo extraído de cada um dos indivíduos do perfil A (In- 01, 03 e 05), enquanto os sesquiterpenos perfizeram $33,8 \%, 29,9 \%$ e $20,5 \%$ deste teor, respectivamente. Os principais monoterpenos foram (Tabela 1): $\beta$-felandreno (In-01, 42,2\%), sabineno (In-01, 4,3 ; In-03, 17,3\%; In-05, 25,1\%) e terpinen-4-ol (In-03, 21,6 ; In-05, 16,2\%). Os sesquiterpenos mais comuns a todos os indivíduos do perfil A foram biciclogermacreno (presente somente em In-01, 20,7\%), espatulenol (In-01 $5,5 \%$; In-03 20,9\%; In-05, 5,1\%) e óxido de cariofileno (In-01, 1\%; In-03, 3,8\%; In-05 7,7\%).

$\mathrm{Na}$ composição do óleo dos indivíduos do perfil B (In- 02, 04 e 06) predominaram sesquiterpenos, correspondendo a 90,5\%, 91,2\% e $96,2 \%$, respectivamente, sendo biciclogermacreno, germacreno-D, trans-cariofileno e espatulenol os principais constituintes (Tabela 1).

Foi possível observar uma boa compatibilidade entre a análise olfativa adotada e os resultados obtidos por CG/EM, ou seja, os indivíduos do perfil A causaram impressões olfativas mais acentuadas (In 01, 03 e 05), que pode ser associado a predominância de monoterpenos, pois nos demais indivíduos, que eram desprovido do odor, observa-se também a ausência de monoterpenos (Minh Tu et al., 2003). A propriedade organoléptica (aroma) está vinculada a ambas classes de constituintes, monoterpenos e sesquiterpenos, no qual este último apresentam um menor número de representantes de real importância na formação de odores básicos (Craveiro, 1988)

As análises por CG/EM permitem, do ponto de vista biossintético, algumas observações. Os monoterpenos encontrados nos indivíduos do perfil A possuem um intermediário em comum, o cátion 4- $\alpha$-terpinil (Dewick, 1995), do qual podem ser gerados os derivados felandrênicos ( $\beta$-felandreno, $42,2 \% \mathrm{em}$ In-01), sabineno (In-01, 4,3\%; In-03, 17,3\%; In-05, $25,1 \%$ ) e terpinen-4-ol (encontrado no In-03, 21,6\%; In-05, 16,2\%). Quanto aos sesquiterpenos desse grupo, constatou-se em In-01 a presença de biciclogermacreno $(20,7 \%)$ e de espatulenol (5,5\%), enquanto em In-03 e In-05 houve somente presença de espatulenol $(20,9 \%$ e $5,1 \%$, respectivamente). Biciclogermacrenoé o precursor de espatulenol, assim como de outros sesquiterpenos, e há alguns relatos da obtenção de espatulenol como um possível artefato, pois o biciclogermacreno se converte facilmente em espatulenol, mas tal conversão ocorre durante o processo de extração e estocagem (Toyota et al., 1996). Nos indivíduos do perfil B predominou somente do grupo germacreno.

Há relatos de variação na composição química de óleos voláteis de diferentes espécies vegetais em função de variações sazonais (Chericoni et al., 2004; Asghari et al., 2002; Taveira et al., 2003; Blank et al., 2007), de latitude (Azevedo et al., 2002) e de condições climáticas (Lago et al., 2003), além de variação relacionada a injúria mecânica (Zabaras \& Wyllie, 2001). Entretanto, uma determinada espécie pode apresentar quimiotipos ou tipos baseados em diferenças genéticas (Thompson et al., 2003) e, conseqüentemente, apresentar polimorfismo químico. A variabilidade química infra-específica já foi documentada em Hyptis suaveolens (Lamiaceae), Ocimum basilicum (Lamiaceae), Melaleuca quinquenervia (Myrtaceae), Thymus vulgaris (Lamiaceae), Thymus mastichina ssp. mastichina (Lamiaceae) (Azevedo et al., 2002; Grayer et al., 1996; Wheeler et al., 2003; Thompson et al., 2003; Miguel et al., 2004; Carvalho-Filho et al., 2006).

No presente trabalho, apesar da grande variabilidade química observada entre os dois grupos, ou seja, a presença principalmnente de monoterpenos, seguida de sequiterpenos, no perfil A e, praticamente, uma ausência de monoterpenos no perfil $B$, juntamente com o número reduzido de indivíduos avaliados (três), bem como a realização de uma só análise para cada individuo, não nos fornece dados estatisticamente suficientes para que se possa discutir a existência de quimiotipos na espécie (Grayer et al., 1996; Rohloff et 
Tabela 1. Compostos presentes no óleo essencial de seis indivíduos de D. furfuracea, coletados em um remanescente de cerrado, em Campo Grande, MS, em outubro de 2004.

\begin{tabular}{|c|c|c|c|c|c|c|}
\hline Indivíduos & \multirow{2}{*}{$01 *$} & \multirow{2}{*}{02} & \multirow{2}{*}{$03 *$} & \multirow{2}{*}{04} & \multirow{2}{*}{$05^{*}$} & \multirow{2}{*}{06} \\
\hline Composto $\boldsymbol{\nabla}$ & & & & & & \\
\hline \multicolumn{7}{|c|}{ Monoterpenos } \\
\hline Sabineno & $4,3 \%$ & - & $17,3 \%$ & - & $25,1 \%$ & - \\
\hline Cis-sabineno & - & - & $1,1 \%$ & - & $1,3 \%$ & - \\
\hline Trans-sabineno & - & - & $1,7 \%$ & - & - & - \\
\hline$\alpha$-Felandreno & $4,6 \%$ & - & $1,2 \%$ & - & - & - \\
\hline$\beta$-Felandreno & $42,2 \%$ & - & $1,2 \%$ & - & $1,5 \%$ & - \\
\hline$\alpha$-Terpineol & - & - & $1,5 \%$ & - & $1,6 \%$ & - \\
\hline Mirceno & $6,8 \%$ & - & - & - & - & - \\
\hline 1-Hidroxi-linalol & - & - & $1,6 \%$ & - & $3,7 \%$ & - \\
\hline Dióxido de limoneno & - & - & $1,1 \%$ & - & $2,9 \%$ & - \\
\hline Terpinen-4-ol & - & - & $21,6 \%$ & - & $16,2 \%$ & - \\
\hline$p$-Cimeno & - & - & $5,6 \%$ & - & $8,3 \%$ & - \\
\hline$\alpha$-Pineno & $3,8 \%$ & - & $1,1 \%$ & - & $3,0 \%$ & $1,5 \%$ \\
\hline$\beta$-Pineno & $2,1 \%$ & - & $1,3 \%$ & - & $2,4 \%$ & $1,5 \%$ \\
\hline Criptona & $1,8 \%$ & - & $1,1 \%$ & - & $2,6 \%$ & - \\
\hline \multicolumn{7}{|c|}{ Sesquiterpenos } \\
\hline Biciclogermacreno & $20,7 \%$ & $21,4 \%$ & - & $24,0 \%$ & - & $29,1 \%$ \\
\hline Espatulenol & $5,5 \%$ & $12,2 \%$ & $20,9 \%$ & $12,4 \%$ & $5,1 \%$ & $18,3 \%$ \\
\hline$\beta$-Elemeno & $0,4 \%$ & $1,7 \%$ & - & $0,6 \%$ & $1,2 \%$ & - \\
\hline Germacreno-D & $1,3 \%$ & $13,6 \%$ & - & $15,0 \%$ & - & $9,6 \%$ \\
\hline Aromadendreno & $0,4 \%$ & - & $0,9 \%$ & $0,7 \%$ & - & $1,7 \%$ \\
\hline$\alpha$-Copaeno & - & $1,5 \%$ & - & $1,5 \%$ & $1,9 \%$ & $1,8 \%$ \\
\hline trans-Cariofileno & $2,0 \%$ & $13,3 \%$ & - & $12,9 \%$ & $1,8 \%$ & $9,3 \%$ \\
\hline$\alpha$-Gurjuneno & - & $1,0 \%$ & - & - & - & - \\
\hline$\gamma$-Muuroleno & - & $1,2 \%$ & - & $0,9 \%$ & - & - \\
\hline Óxido de cariofileno & $1,0 \%$ & $5,2 \%$ & $3,8 \%$ & $6,8 \%$ & $7,7 \%$ & $5,3 \%$ \\
\hline$\Delta$-Cadineno & $1,4 \%$ & $1,7 \%$ & - & $1,7 \%$ & - & $5,5 \%$ \\
\hline$\beta$-Selineno & - & $1,1 \%$ & - & - & - & $1,5 \%$ \\
\hline$\alpha$-Humuleno & $0,6 \%$ & $3,3 \%$ & - & $2,6 \%$ & - & $1,9 \%$ \\
\hline Isoespatulenol & - & $2,2 \%$ & - & $1,1 \%$ & - & $1,6 \%$ \\
\hline Ledol & - & $1,1 \%$ & $0,8 \%$ & $1,3 \%$ & - & - \\
\hline$\alpha$-Cadinol & - & $2,8 \%$ & - & $2,9 \%$ & - & $1,8 \%$ \\
\hline$\Delta$-Cadinol & - & $1,2 \%$ & - & $1,1 \%$ & - & $1,6 \%$ \\
\hline$\beta$-Copaen-4- $\alpha$-ol & - & $1,7 \%$ & $1,1 \%$ & $0,9 \%$ & - & $1,6 \%$ \\
\hline Epi- $\alpha$-cadinol & - & $2,0 \%$ & - & - & - & $2,3 \%$ \\
\hline Viridiflorol & $0,5 \%$ & $2,3 \%$ & $1,6 \%$ & $3,1 \%$ & - & $3,3 \%$ \\
\hline$\alpha$-Tujeno & - & - & $0,8 \%$ & - & $2,8 \%$ & - \\
\hline$\Delta$-Guaieno & - & - & - & $1,7 \%$ & - & - \\
\hline Não identificados & - & $9,5 \%$ & $12,4 \%$ & $8,8 \%$ & $10,6 \%$ & - \\
\hline Total & $99,4 \%$ & $100,0 \%$ & $99,7 \%$ & $100 \%$ & $99,7 \%$ & $99,2 \%$ \\
\hline
\end{tabular}

(*) Indivíduos de perfil A. 
al., 2004). Mas é possível afirmar, que a varibilidade aqui registrada parece não estar relacionada com fatores ambientais ou com caracteres morfológicos, e tampouco com o estado fenológico dos indivíduos (Tabela 2), visto que In-03, 04, 05 e 06 estavam no período de floração, o que não ocorria com In-01 e 02. Embora não se tenham encontrado estudos sobre a influência de galhas na composição do óleo essencial, sua presença em In-01, 02, 03 e 05 aparentemente não afetou a composição desse óleo. A presença de galhas do tipo globóide verde em $D$. furfuracea fora anteriormente documentada (Scarelli-Santos \& Varanda, 2001).

Pode-se ainda tecer comentários comparativos a outras espécies de Duguetia presentes em regiões distintas a essa coletada no cerrado. A composição do óleo essencial de 5 espécies de Duguetia da região amazônica (D. eximia, D. flagellaris, D. pycnastera, D. riparia e $D$. trunciflora) foi analisada e apresentou predominância de sesquiterpenos oxigenados, sendo o principal constituinte o espatulenol. Algumas espécies de Xylopia da região amazônica também mostram a predominância de sesquiterpenos, sendo o espatulenol predominante em $X$. aromatica e $X$. emarginata. A diferença entre a composição química de espécies de Duguetia, bem como de Xylopia, coletadas nas regiões amazônica e cerrado tem sido acompanhada e analisada, visando avaliar as possíveis implicações quimiotaxonômicas que possam ocorrer em função das diferenças climáticas existentes (Maia et al., 2006, Maia et al., 2005, Lago et al., 2003; Fechine et al., 2002). Tem-se sugerido a predominância de constituintes oxigenados nos óleos essenciais de espécies de Annonaceae coletadas na região amazônica, quando comparada com as do cerrado (Lago et al., 2003). Nos indivíduos aqui analisados, observa-se a predominância de biciclogermacreno e germacreno $\mathrm{D}$, enquanto que os oxigenados, como o espatulenol, está presente em maior quantidade somente no In 03 , que predomina monoterpenos.

Tabela 2. Caracteres morfológicos analisados em seis indivíduos de D. furfuracea de um remanescente de cerrado em Campo Grande - MS.

\begin{tabular}{|c|c|c|c|c|c|c|c|c|c|}
\hline & \multicolumn{3}{|c|}{$\begin{array}{l}\text { Indivíduos } \\
\text { Caracteres } \nabla\end{array}$} & In 01 & In 02 & In 03 & In 04 & In 05 & In 06 \\
\hline \multirow[t]{4}{*}{ Hábito } & \multicolumn{3}{|l|}{ Altura } & $0,6 \mathrm{~m}$ & $1,5 \mathrm{~m}$ & $1,0 \mathrm{~m}$ & $0,6 \mathrm{~m}$ & $0,6 \mathrm{~m}$ & $1,5 \mathrm{~m}$ \\
\hline & \multicolumn{3}{|l|}{ Odor } & presente & ausente & presente & ausente & presente & ausente \\
\hline & \multicolumn{3}{|c|}{ Comprimento / Largura $(\mathrm{cm})$} & $\begin{array}{c}15 \text { a } 18 \mathrm{~cm} / \\
5 \text { a } 6 \mathrm{~cm}\end{array}$ & $\begin{array}{c}12 \text { a } 14 \mathrm{~cm} / \\
4 \text { a } 5 \mathrm{~cm}\end{array}$ & $\begin{array}{c}14 \text { a } 18 \mathrm{~cm} / \\
4,5 \text { a } 6 \mathrm{~cm}\end{array}$ & $\begin{array}{l}11 \text { a } 13 \mathrm{~cm} / \\
3,5 \text { a } 4,5 \mathrm{~cm}\end{array}$ & $\begin{array}{l}12 \text { a } 13 \mathrm{~cm} / \\
4 \text { a } 4,5 \mathrm{~cm}\end{array}$ & $\begin{array}{c}12,5 \text { a } 14,5 \mathrm{~cm} / \\
4 \text { a } 4,5 \mathrm{~cm}\end{array}$ \\
\hline & \multirow{3}{*}{$\begin{array}{c}\text { Face } \\
\text { Adaxial }\end{array}$} & \multicolumn{2}{|c|}{ Tricomas } & estrelados & estrelados & estrelados & estrelados & estrelados & estrelados \\
\hline \multirow{3}{*}{ Folha } & & & Forma & globóide & globóide & achatada & - & globóide & - \\
\hline & & Ualnas & Coloração & verde & verde & acinzentada & - & verde & - \\
\hline & $\begin{array}{c}\text { Face } \\
\text { Abaxial }\end{array}$ & \multicolumn{2}{|c|}{ Tricomas } & escamosos & escamosos & escamosos & escamosos & escamosos & escamosos \\
\hline \multirow{4}{*}{ Flores } & \multirow{2}{*}{ Sépala } & \multicolumn{2}{|c|}{ Tamanho $(\mathrm{cm})$} & - & - & $1 \mathrm{~cm}$ & $1,5 \mathrm{~cm}$ & $1,5 \mathrm{~cm}$ & $1,3 \mathrm{~cm}$ \\
\hline & & Coloraç: & & - & - & verde & verde & verde & verde \\
\hline & \multirow{2}{*}{ Pétala } & \multirow{2}{*}{\multicolumn{2}{|c|}{$\begin{array}{l}\text { Tamanho }(\mathrm{cm}) \\
\text { Coloração }\end{array}$}} & - & - & 1,8 a $2,2 \mathrm{~cm}$ & 2,1 a $2,5 \mathrm{~cm}$ & 2,2 a $2,3 \mathrm{~cm}$ & 2 a $2,5 \mathrm{~cm}$ \\
\hline & & & & - & - & $\begin{array}{c}\text { rosa } \\
\text { avermelhada }\end{array}$ & $\begin{array}{c}\text { rosa } \\
\text { avermelhada }\end{array}$ & $\begin{array}{c}\text { rosa } \\
\text { avermelhada }\end{array}$ & $\begin{array}{c}\text { rosa } \\
\text { avermelhada }\end{array}$ \\
\hline
\end{tabular}

—: ausente Obs. Não foi observado odor nos frutos e flores, bem como estavam ausentes a presença de galhas coloração na face abaxial das folhas.

\section{AGRADECIMENTOS}

À Fundação de Apoio ao Desenvolvimento do Ensino, Ciência e Tecnologia do Estado de Mato Grosso do Sul (FUNDECT), pelo financiamento deste projeto.

\section{REFERÊNCIAS}

Adams RP 1995. Identification of essential oil components by gas chromatography/mass spectroscopy. Ilinois: Allured Publishing Corporation.

Agra MF, França PF, Barbosa-Filho JM 2007. Synopsis of the plants known as medicinal and poisonous in
Northeast of Brazil. Rev Bras Farmacogn 17: 114140.

Asghari G, Houshfar G, Mahmaoudi Z 2002. Seasonal variation of mono- and sesquiterpenes in the essential oil of Pycnocycla spinosa. Iran J Pharm Res 1: 61-63.

Azevedo NR, Campos IFP, Ferreira HD, Portes TA, Seraphin JC, Paula JR, Santos SC, Ferri PH 2002. Essential oil chemotypes in Hyptis suaveolens from Brazilian Cerrado. Bioch Syst Ecol 30: 205-216.

Barbosa-Filho JM, Cunha RM, Dias CS, Athayde-Filho PF, Silva MS, Cunha EVL, Machado MIL, Craveiro AA, Medeiros IA 2008. GC-MS Analysis and cardiovascular activity of the essential oil of Ocotea duckei. Rev Bras Farmacogn 18: 37-41. 
Blank AF, Costa AG, Arrigoni-Blank MF, Cavalcanti SCH, Alves PB, Innecco R, Ehlert PAD, Sousa IF 2007. Influence of season, harvest time and drying on Java citronella (Cymbopogon winterianus Jowitt) volatile oil. Rev Bras Farmacogn 17: 557-564.

Carollo CA, Hellmann AR, Siqueira JM 2005. Sesquiterpenoids from volatile oil from leaves of Duguetia furfuracea (Annonaceae). Bioch Syst Ecol 33: 647-649.

Carollo CA, Hellmann-Carollo AR, Siqueira JM, Albuquerque S 2006a. Alkaloids and a flavonoid from aerial parts (leaves and twigs) of Duguetia furfuracea Annonaceae. J Chil Chem Soc 51: 837-841.

Carollo CA, Siqueira JM, Garcez WS, Diniz R, Fernandez NG. 2006b. $N$-Nitrosoanonaine and $N$-nitrosoxylopine, aporphine alkaloids from Duguetia furfuracea. J Nat Prod 69: 222-224.

Carvalho-Filho JLS, Blank AF, Alves PB, Ehlert PAD, Melo AS, Cavalcanti SCH, Arrigoni-Blank MF, SilvaMann R 2006. Influence of the harvesting time, temperature and drying period on basil (Ocimum basilicum L.) essential oil. Rev Bras Farmacogn 16: 24-30.

Chericoni S, Flamini G, Campeol E, Cioni PL, Morelli I 2004. GC-MS analyses of the essential oil from the aerial parts of Artemisia verlotiorum: variability during the year. Bioch Syst Ecol 32: 423-429.

Craveiro AA 1988. De aromas, de insetos e de plantas. In: Gottlieb, O. R. (org.) Perspectivas da química de produtos naturais. Publicação ACIESP, n.45-III, p.107-147.

Da Silva Coelho L 2003. Medicinal plant based renal colic treatment agent consists of 250 milligramme capsules based on Duguetia furfuracea material. Dervewent Innovations Index Br20022030-A.

Dewick PM 1995. The biosynthesis of $\mathrm{C}_{5}-\mathrm{C}_{20}$ terpenoid compounds. Nat Prod Rep 12: 507-534.

Fechine IM, Navarro VR, Cunha EVL, Silva MS, Maia JGS, Barbosa-Filho JM 2002. Alkaloids and volatile constituents from Duguetia flagellaris. Bioch Syst Ecol 30: 267-269.

Franco MRB, Rodriguez-Amaya D, Lanças FM 2004. Compostos voláteis de três cultivares de manga (Mangifera indica L.). Cien Tec Alim 24: 165-169.

Grayer RJ, Kite GC, Goldstone FJ, Bryan SE, Paton A, Putievsky E 1996. Infraspecific taxonomy and essential oil chemotypes in sweet basil, Ocimum basilicum. Phytochemistry 43: 1033-1039.

Grosch W 1998. Detection of potent odorants in foods bu aroma extract dilution analysis. Trends Food Sci Tech 4: 68-73.

Grosch W 2001. Evaluation of the key odorants of foods by dilution experiments, aroma models and omission Chem Sense 26: 533-545.

Harris JG, Harris MW 1994. Plant identification terminology: An illustrated glossary, Payson, Utah: Spring Lake Publishing.

Lago JHG, Júnior PA, Moreno PRH, Limberger RP, Apel MA, Henriques AT 2003. Analysis, comparison and variation on the chemical composition from the leaf volatile oil of Xylopia aromatica (Annonaceae). Bioch Syst Ecol 31: 669-762.

Lorenzi H 2000. Plantas daninhas do Brasil: terrestres, aquáticas, parasitas e tóxicas. Nova Odessa: Instituto Plantarum de Estudos da Flora Ltda.

Maas PJM, Kamer HM, Junikka L, Mello-Silva R, Rainer
H 2001. Annonaceae from Central-Eastern Brazil. Rodriguésia 52: 65-98.

Maia JGS, Andrade EHA, Silva AC, Oliveira J, Carreira LMM, Araújo JS 2005. Leaf volatile oils from four Brazilian Xylopia species. Flav Frag J.20: 474-747.

Maia JGS, Andrade EHA, Carreira LMM, Oliveira J 2006. Essential oil composition from Duguetia species (Annonaceae). J Essent Oil Res 18: 60-63.

Miguel MG, Duarte FL, Venâncio F, Tavares R 2004. Comparison of the main components of the essential oils from flowers and leaves of Thymus mastichina (L.) L. ssp. mastichina collected at different regions of Portugal. J Essent Oil Res 16: 323-327.

Minh Tu NT, Onishi Y, Son US, Ogawa E, Ukeda H, Sawamura M 2003. Characteristic odour components of Citrus inflate Hort. Ex Tank (Mochiyu) cold-pressed peel oil. Flav Frag J 18: 454-459.

Ngassoum MB, Ousmaila H, Ngamo LT, Maponmetsem PM, Jirovetz L, Buchbauer G 2004. Aroma compounds of essential oils of two varieties of the spice plant Ocimum canum Sims from northern Cameroon. $J$ Food Comp Anal 17: 197-204.

Pott A, Pott VJ 1994. Plantas do Pantanal. Corumbá: Embrapa-Spi.

Rodrigues VEG, Carvalho DA 2001. Levantamento etnobotânico de plantas medicinais no domínio cerrado na região do alto Rio Grande - Minas Gerais Cienc Agrotec 25: 102-123.

Rohloff J, Mordal R, Dragland S 2004. Chemotypical variation of Tansy (Tanacetum vulgare L.) from 40 different locations in Norway. J Agric Food Chem 52: 17421748.

Santos DYAC, Salatino MLF 2000. Foliar flavonoids of Annonaceae from Brazil: taxonomic significance. Phytochemistry 55: 567-573.

Scarelli-Santos C, Varanda EM 2001. Morfologia de galhas foliares de Duguetia furfuracea (A. St.-Hil.) Benth. $\&$ Hook. f. (Annonaceae). Resumos do $52^{\circ}$ Congresso Nacional de Botânica, João Pessoa.

Silberbauer-Gottsberger I 1981/1982. O cerrado como potencial de plantas medicinais e tóxicas. Oréades 14/15: 15-30.

Taveira FSN, Andrade EHA, Lima WN, Maia JGS 2003. Seasonal variation in the essential oil of Pilocarpus microphyllus Stapf. An Acad Bras Cienc 75: 27-31.

Thompson JD, Chalchat JC, Michet A, Linhart YB, Ehlers B 2003. Qualitative and quantitative variation in monoterpene co-occurrence and composition in the essential oil of Thymus vulgaris chemotypes. J Chem Ecol 29: 859-880.

Toyota M, Koyama H, Mizutani M, Asakawa Y 1996. (-)Ent-spathulenol isolated from liverworts is a artefact Phytochemistry 41: 1347-1350.

Wheeler GS, Massey LM, Southwell IA 2003. Dietary influences on terpenoids sequestered by the biological control agent Oxyops vitiosa: effect of plant volatiles from different Melaleuca quinquenervia chemotypes and laboratory host species. J Chem Ecol 29: 189208.

Zabaras D, Wyllie SG 2001. The effect of mechanical wounding on the composition of essential oil from Ocimum minimum L. leaves. Molecules 6: 79-86. 